Abstract

\title{
Integrating Crop Modelling, Physiology, Genetics and Breeding to Aid Crop Improvement for Changing Environments in the Australian Wheatbelt ${ }^{+}$
}

\author{
Karine Chenu*, Andrew Fletcher, Behnam Ababaei, Jack Christopher, Alison Kelly, Lee Hickey, \\ Erik Van Oosterom and Graeme Hammer \\ The University of Queensland, Queensland Alliance for Agriculture and Food Innovation (QAAFI), \\ Toowoomba, QLD 4350, Australia; andrew.fletcher1@uqconnect.edu.au (A.F.); b.ababaei@uq.edu.au (B.A.); \\ j.christopher@uq.edu.au (J.C.); a.kelly1@uq.edu.au (A.K.); 1.hickey@uq.edu.au (L.H.); \\ erik.van.oosterom@uq.edu.au (E.V.O.); g.hammer@uq.edu.au (G.H.) \\ * Correspondence: karine.chenu@uq.edu.au \\ + Presented at the third International Tropical Agriculture Conference (TROPAG 2019), Brisbane, Australia, \\ 11-13 November 2019.
}

Published: 24 December 2019

\begin{abstract}
Despite recent progress in genetics, genomics, and phenotyping, trait selection is limited by our ability to predict genotype $\mathrm{x}$ environment interactions, and to identify impactful traits for target environments. Here, we combined crop modelling, physiology, genetics, and breeding to identify relevant traits to increase wheat yields in the target environments of the Australian wheatbelt, develop high-throughput phenotyping methods for relevant traits, and identify their underlying genetic controls. Better transpiration efficiency (i.e., 'more crop per drop') and its components were identified as traits of interest and used as an example to illustrate how integrating modelling, phenotyping, and genetics can be used to improve crop adaptation. We anticipate that such an approach can enhance the efficiency of breeding programmes to increase productivity in target environments in current and future climates.
\end{abstract}

Keywords: crop adaptation; drought; transpiration efficiency; wheat; APSIM

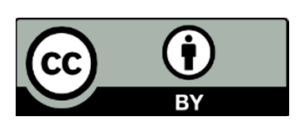

(C) 2019 by the authors. Licensee MDPI, Basel, Switzerland. This article is an open access article distributed under the terms and conditions of the Creative Commons Attribution (CC BY) license (http://creativecommons.org/licenses/by/4.0/). 